\title{
Características del cibario y órganos genitales internos en la identificación de Lutzomyia longipalpis hembra
}

\author{
Llano, E.G.; Maidana, H.R.; Cabrera, W.R.; Báez, A.D.; Meyer, S.N.
}

Servicio de Diagnóstico de Leishmaniosis, Hospital de Clínicas, Facultad de Ciencias Veterinarias, Universidad Nacional del Nordeste, Sargento Cabral 2139, Corrientes (3400), Argentina. Tel: 379-4425753.

E-mail: anato2@vet.unne.edu.ar.

\begin{abstract}
Resumen
Llano, E.G.; Maidana, H.R.; Cabrera, W.R.; Báez, A.D.; Meyer, S.N.: Características del cibario y órganos genitales internos en la identificación de Lutzomyia longipalpis hembra. Rev. vet. 25: 1, 61-63, 2014. El control epidemiológico de la leishmaniosis canina requiere establecer fehacientemente la existencia del vector de la enfermedad. El flebótomo Lutzomyia longipalpis es el transmisor de la leishmaniosis visceral del perro. Dado que en el nordeste argentino existen otras especies de flebótomos del mismo género, la presente investigación tuvo como objetivo verificar la eficacia de algunos descriptores anatómicos microscópicos para constatar la presencia de hembras de L. longipalpis. De los 63 descriptores aptos para este fin, se seleccionaron algunos ubicados en la cabeza del insecto (cibario y antena) y en la región abdominal caudal (genitales internos). Estos descriptores se revelaron como los más relevantes y factibles de ser distinguidos, permitiendo una rápida y segura identificación del flebótomo. Se espera que esta información resulte útil para los encargados del control epizootiológico de la leishmaniosis.
\end{abstract}

Palabras clave: Lutzomyia longipalpis, identificación, leishmaniosis, vector.

\begin{abstract}
Llano, E.G.; Maidana, H.R.; Cabrera, W.R.; Báez, A.D.; Meyer, S.N.: Characteristics of the cibarial and internal genital organs for the identification of female Lutzomyia longipalpis. Rev. vet. 25: 1, 61-63, 2014. Canine leishmaniasis epidemic control requires to accurately establish the existence of the vector for a considered region. The phlebotomus Lutzomyia longipalpis is the transmitter of the canine visceral leishmaniasis. Since other species of phlebotomus of the same genus exist in Northeastern Argentina, the aim of this investigation was to verify the effectiveness of some microscopic anatomic descriptors to verify the presence of females L. longipalpis. From 63 descriptors available for this purpose, some regarding the head (cibario and antenna) and low abdominal region (internal genital) of the insect, were selected. These descriptors are the most outstanding and feasible, allowing a quick and accurate identification of the phlebotomus. This information might be useful for leishmaniasis epizoothiological control.
\end{abstract}

Key words: Lutzomyia longipalpis, identification, leishmaniosis, vector.

\section{INTRODUCCIÓN}

Una reciente investigación realizada en la Provincia de Corrientes sobre el género Lutzomya reveló la existencia de las especies $L$. longipalpis $(86,3 \%$, vector de leishmaniosis visceral), $L$. neivai $(10,1 \%$, vector de leishmaniosis tegumentaria), así como L. migonei $(2,5 \%)$ y $L$. cortelezzii-sallesi $(0,7 \%)$, vectores secundarios de leishmaniosis cutánea ${ }^{7}$. En Argentina se ha reportado un total de 28 especies de la subfamilia Phle-

Recibido: 12 febrero 2014 / Aceptado: 14 marzo 2014 botominae (Diptera: Psychodidae), la mayoría de ellas pertenecientes al género Lutzomyia ${ }^{8}$.

El ciclo de vida de estos insectos abarca una metamorfosis a través de los estadios de huevo, larva, pupa y adulto que dura de 30 a 100 días dependiendo de la especie y condiciones ambientales. Los tres primeros estadios son difíciles de observar en su ambiente natural. Los adultos son pequeños (2-3 mm), con la cabeza situada en ángulo de $90^{\circ}$ con respecto al abdomen, alas lanceoladas (erectas en "V" durante el reposo) y coloración que varía desde el gris al marrón claro u oscuro. El insecto, cubierto por una pilosidad densa e hirsuta, 
posee patas y antenas largas y delgadas. Son voladores débiles y silenciosos; su radio de dispersión rara vez sobrepasa los 200 metros. La mayor actividad la realizan al atardecer y amanecer o durante el día cuando está muy nublado. Se refugian del sol directo, viento y lluvia en huecos de árboles, cuevas de animales y otros sitios oscuros con condiciones de temperatura y humedad estable, alimentándose de jugos azucarados que encuentran en la savia de plantas, frutos maduros y áfidos ("pulgones"). La hembra necesita ingerir sangre para el desarrollo de los huevos y lo hace cortando y exponiendo capilares superficiales de la piel (telmofagia) de un amplio rango de hospederos vertebrados incluyendo aves, mamíferos y reptiles ${ }^{3,5,6}$.

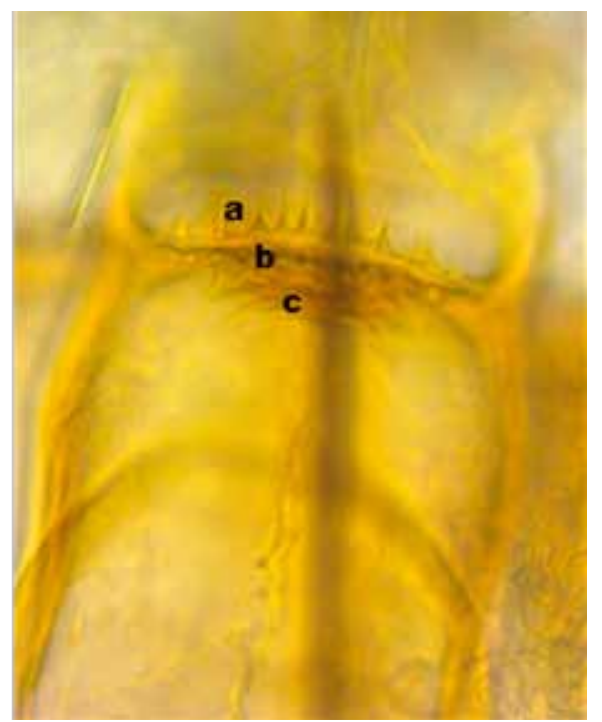

Figura 1. Cibario de L. longipalpis 40x. a) dientes horizontales, b) dientes verticales, c) área pigmentada.

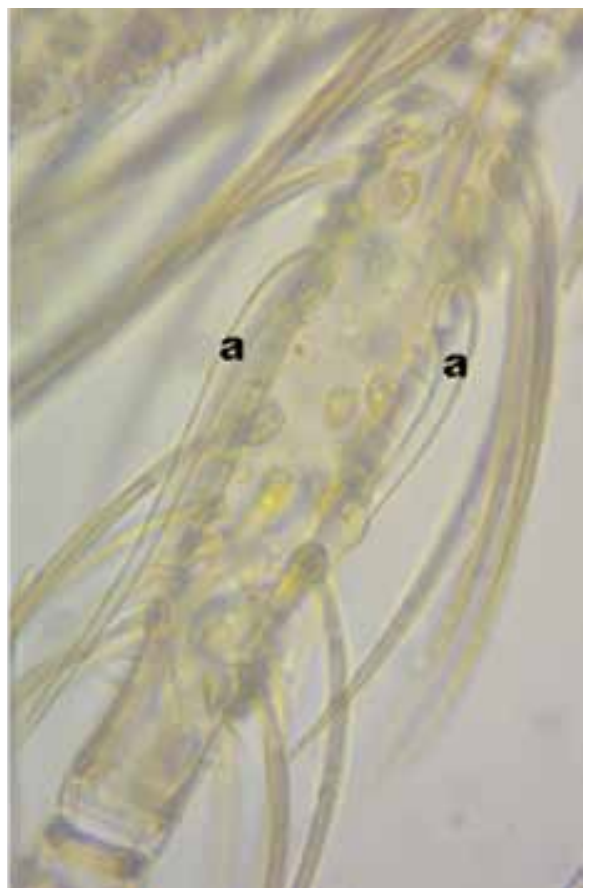

Figura 2. Flagelómero II de L. longipalpis 100x. a) ascoide.
Existen diversos métodos para efectuar la captura de Lutzomyia sp., que varían según el objetivo perseguido en la investigación (tubos aspiradores y trampas con cebos atrayentes o luminosos, así como adhesivos de interferencia de vuelo) ${ }^{10}$. Los insectos capturados se sacrifican en una cámara cerrada conteniendo éter, cloroformo o acetato de etilo. Para observar al microscopio los órganos internos, se debe efectuar el aclaramiento de los insectos empleando soluciones de $\mathrm{NaOH}$ o KOH al 10-20\%, así como lactofenol (ácido láctico y fenol 1:1) o bien detergente diluido al 5\%. El tiempo de aclaramiento varía desde algunas horas hasta varios días ${ }^{5,9}$.

Para identificar microscópicamente ejemplares de L. longipalpis de sexo hembra, existen aproximadamente 63 descriptores. A nivel de la cabeza adquiere importancia el cibario (segmento del aparato digestivo situado oralmente a la faringe) donde se puede observar el número y forma de los dientes horizontales posteriores, las hileras de dientes verticales anteriores y la forma y extensión oral del área pigmentada. En el segundo segmento antenal o flagelómero II es relevante verificar la extensión de la prolongación distal de los ascoides (estructuras pares de diámetro mayor que el de los pelos que se implantan equidistantes en la superficie de los flagelómeros). En los órganos genitales internos asumen importancia el aspecto del cuerpo de las espermatecas (segmentos pares del aparato genital con función de depósito de espermatozoides), así como la longitud y el ancho de los ductos genitales individuales ${ }^{1,2}$.

Habida cuenta que en ocasiones anteriores nos ocupamos de la identificación rápida de L. longipalpis macho (Rev. vet. 24: 1, 21-24, 2013), el objetivo de la presente comunicación fue difundir los descriptores útiles para reconocer la hembra de dicha especie, empleando para ello las características del cibario y los genitales internos, los cuales permiten una rápida ubicación taxonómica para diferenciarla de otros flebótomos presentes en la región.

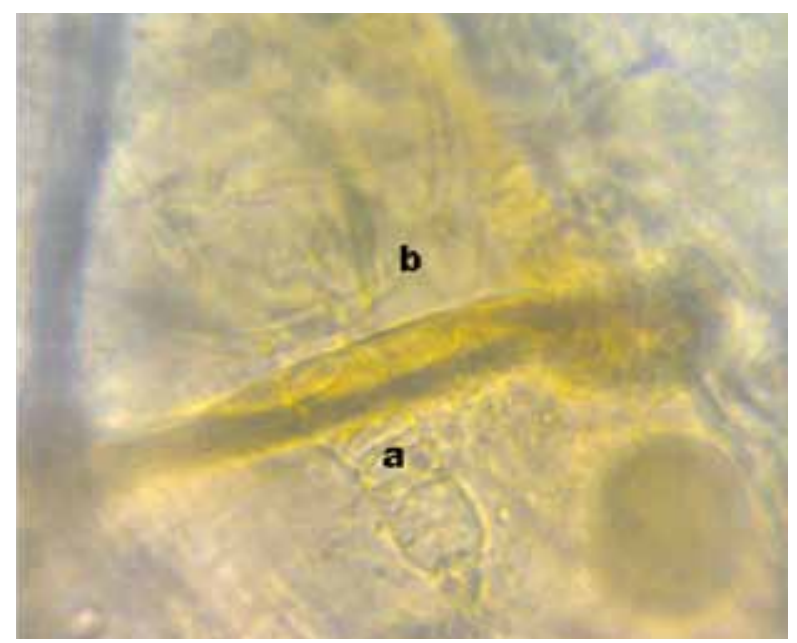

Figura 3. Genitales internos de L. longipalpis 100x. a) cuerpo de espermateca, b) ducto genital individual. 


\section{MATERIAL Y MÉTODOS}

Para la captura y procesamiento de los flebótomos se utilizaron los mismos elementos y procedimientos descriptos en la publicación precitada. Se empleó una trampa de luz y los ejemplares se sacrificaron por frío (freezer), tras lo cual se "aclararon" con $\mathrm{KOH} \mathrm{10 \%} \mathrm{du-}$ rante $24-48 \mathrm{~h}$, se lavaron con etanol $70 \%$ y se montaron en portaobjetos con aceite de inmersión, examinándolos con objetivo de inmersión. Las imágenes de interés fueron fotografiadas con una cámara digital fijada a un microscopio y luego procesadas por el programa Axio Vision Rel 4.8.

\section{RESULTADOS Y DISCUSIÓN}

El 73\% de los especimenes capturados fueron identificados como L. longipalpis, en una relación 3:1 entre machos y hembras respectivamente. De las observaciones microscópicas para la identificación de hembras de L. longipalpis surgió la conveniencia de utilizar algunos descriptores a nivel de la cabeza, principalmente estructuras situadas en el cibario, como así también descriptores de los órganos genitales internos, alojados en el abdomen caudal.

Los descriptores de la cabeza comprendieron 8 a 10 dientes horizontales posteriores ubicados en el cibario (Figura 1, a), una hilera de dientes verticales anteriores, también en el cibario (Figura 1, b), un área pigmentada del cibario en forma de meseta con leve convexidad oral (Figura 1, c) y la extensión de los ascoides que alcanzaban o sobrepasaban la extremidad distal del flagelómero (Figura 2).

Los descriptores ubicados en los genitales internos de la región del abdomen caudal incluyeron el aspecto plegado del cuerpo de las espermatecas (Figura 3, a) y la presencia de ductos genitales individuales largos y de ancho uniforme (Figura 3, b).

La identificación de las hembras de L. longipalpis adquiere importancia porque debido a su conducta alimentaria hematófaga actúa como vector, constituyendo uno de los eslabones indispensables en la cadena epidemiológica de la leishmaniosis visceral ${ }^{4}$. Además de verificar su existencia, con técnicas ad hoc es posible confirmar su infección natural con Leishmania chagasi.

Los descriptores mencionados ${ }^{1}$ fueron seleccionados por ser los más rápidos y sencillos, permitiendo que operadores regionales de la salud pública puedan identificar el insecto en estudio sin necesidad de poseer altos estudios entomológicos.

\section{REFERENCIAS}

1. Fernandes PH, Tolezano JE, Bianchi EA. 2011. Chave de identificação ilustrada dos Phlebotominae (Diptera, Psychodidae) do estado de São Paulo, Brasil. Papéis Avulsos Zool 51: 405-409.

2. Laboratoire Informatique et Systématique (LIS, Paris). 2009. Base de datos de flebótomos de las Américas. On line: lis-upmc.snv.jussieu.fr/xper2/basesHtml/phle- botominae_es/web//index.html.

3. Lucientes J, Astillo JA, Gracia MJ, Peribañez MA. 2005. Flebótomos: de la biología al control. Rev Elec Vet RedVet. http://www.veterinaria.org/revistas/redvet.

4. Ministerio de la Protección Social, Instituto Nacional de Salud (Colombia). 2011. Gestión para la vigilancia entomológica y control de la transmisión de leishmaniasis. Med \& Lab 1: 53-54.

5. Programa de estudio y control de enfermedades tropicales (PECET). 2010. Manual de diagnóstico y control de la leishmaniasis en Centroamérica, Publ. Universidad de Antioquia, Medellín (Colombia), p. 64-67.

6. Salomon OD. 2005. Phlebotominae-Flebótomos, Publ. Fundación Mundo Sano (Buenos Aires) 10: 67-73.

7. Salomon OD, Ramos LK, Quintana MG, Acardi SA, Santini MS, Schneider A. 2009. Distribución de vectores de Leishmaniasis visceral en la Provincia de Corrientes. Medicina (Buenos Aires) 69: 625-630.

8. Salomon OD, Andrade JD, Fernandez MS, Rosa JR, Szelag EA, Santini MS. 2010. Nuevos registros de Phlebotominae (Diptera: Psychodidae) para la Argentina. Rev Soc Entomol Argent 69: 3-4.

9. Torres Gutiérrez C. 2009. Manual para el estudio e identificación de vectores de leishmaniasis. Documento guía del Curso Latinoamericano de Estudio e Identificación de vectores de leishmaniasis (OMS-OPS-PECET), Medellín (Colombia), p. 52-54.

10. Vivero RJ. 2009. Guía fotográfica de flebotomíneos presentes en Colombia. Métodos de colecta. Publ. Programa de Estudio y Control de Enfermedades Tropicales (PECET), Universidad de Antioquia (Medellín, Colombia), p. 9. 